\title{
Relative Risk of Anxiety and Depression among COVID-19 Survivor Healthcare Workers from a Tertiary Care Hospital in Pakistan: A Pilot Cohort Study
}

\author{
Farnaz Zahoor ${ }^{1}$, Zahid Nazar ${ }^{2}$, Sarwat Masud ${ }^{3}$ and Rehana Rahim ${ }^{1}$ \\ ${ }^{1}$ Department of Obstetrics \& Gynecology, Lady Reading Hospital, Peshawar, Khyber Pakhtunkhwa, Pakistan \\ ${ }^{2}$ Department of Psychiatry, Lady Reading Hospital, Peshawar, Khyber Pakhtunkhwa, Pakistan \\ ${ }^{3}$ Department of Emergency, The Aga Khan University Hospital, Karachi, Pakistan
}

\begin{abstract}
Longitudinal studies for determining the risk of anxiety and depression among COVID-19 survivor healthcare workers are lacking. This study aimed to determine the risk of anxiety and depression among healthcare workers that survived COVID-19 infection through a six-month post-recovery follow-up. This was a cohort study conducted at the Department of Obstetrics and Gynecology, Lady Reading Hospital, Peshawar, from 1st March to $30^{\text {th }}$ August 2020. A total of 64 subjects participated. Hospital Anxiety and Depression Scale (HADS) was used for assessing the anxiety and depression symptoms. HADS has three score_cutoff as_normal, borderline abnormal and, abnormal anxiety/depression. For the purpose of the study, borderline and above scores were taken as positive for anxiety/depression. Data was analysed using STATA Corp 15.1. There was no increased risk of anxiety or depression six months post-COVID-19 infection in the infected HCW, compared to uninfected HCW. It is recommended to conduct longitudinal studies with large sample and longer follow-up.
\end{abstract}

Key Words: Relative risk, Anxiety, Depression, HADS, COVID-19, Healthcare workers.

How to cite this article: Zahoor F, Nazar Z, Masud S, Rahim R. Relative Risk of Anxiety and Depression among COVID-19 Survivor Healthcare Workers from a Tertiary Care Hospital in Pakistan: A Pilot Cohort Study. J Coll Physicians Surg Pak 2021; 31(10):1244-1246.

The impact of COVID-19 on mental health of healthcare workers (HCWs) is a public health issue of concern. It is predicted that the pandemic will have both short-term and long-term consequences on the mental health of people. ${ }^{1}$ Most of the current evidence about the impact of COVID-19 on mental health is based on cross-sectional studies and very few longitudinal studies are found. Mazza et al. hypothesised that COVID-19 survivors have a high prevalence of psychiatric illness after recovery, compared to the general population. Four thousand patients were screened one month after hospitalisation for COVID-19, and found high rate of anxiety, depression, and PTSD. ${ }^{2}$ One local study reported increased stress among HCWs in Peshawar. ${ }^{3}$ However, studies that determine the risk of anxiety and depression among the COVID-19 survivor HCWs are lacking.

Correspondence to: Dr. Farnaz Zahoor, Department of Obstetrics \& Gynecology, Lady Reading Hospital, Peshawar, Khyber Pakhtunkhwa, Pakistan

E-mail: farnazzahoor1410@hotmail.com

Received: October 24, 2020; Revised: July 06, 2021;

Accepted: July 14, 2021

DOI: https://doi.org/10.29271/jcpsp.2021.10.1244
This study aimed to determine the risk of anxiety and depression among HCWs six months post-COVID-19 infection using HADS screening and diagnostic instruments for detection of anxiety and depression. The primary outcome of this study was to determine the relative risk of anxiety and depression among HCWs infected with COVID-19, compared to those that were uninfected with COVID-19.

This was a cohort study conducted at the Department of Obstetrics and Gynecology, Lady Reading Hospital, from 1st March to $30^{\text {th }}$ August 2020. Data were collected from HCWs (residents, interns, consultants, nurses and, ancillary staff). All HCWs COVID-19 PCR positive in March 2020, were taken as the exposed group and all HCWs COVID-19 negative in March 2020 were enrolled as the unexposed group. HCWs with a prior history of diagnosed mental illness or on psychiatric medications were excluded. Data were collected prospectively. Both the exposed and unexposed groups were recruited from the same unit to minimise confounders. Previous pandemics have shown a $10-35 \%$ risk for developing anxiety and depression post-infection. ${ }^{2}$ Taking a margin of error of $5 \%$ and confidence level of $95 \%$, risk of anxiety and depression of $30 \%$ among exposed, and $5 \%$ among the unexposed, WHO calculator was used to calculate the sample size to be 43 among exposed and 43 among unexposed group. Aconvenience sampling technique wasused. 
Table I: Association of anxiety and depression with demographic variables.

\begin{tabular}{|c|c|c|c|c|c|c|c|c|}
\hline Demographics & $\begin{array}{c}\text { Anxiety } \\
\text { positive } \\
n=38(\%)\end{array}$ & $\begin{array}{c}\text { Anxiety } \\
\text { negative } \\
n=26 \text { (\%) }\end{array}$ & p-value & $+\mathbf{R R}$ & $\begin{array}{c}\text { Depression } \\
\text { positive } \\
n=41(\%)\end{array}$ & $\begin{array}{c}\text { Depression } \\
\text { negative } \\
n=23(\%)\end{array}$ & p-value & $+\mathbf{R R}$ \\
\hline \multicolumn{3}{|l|}{ Age } & 0.847 & 0.94 & & & 0.491 & 0.83 \\
\hline$<35$ & $33^{\mathrm{a}}(86.8)$ & $23^{\mathrm{b}}(88.5)$ & & & $35^{\mathrm{a}}(85.4)$ & $21^{b}(91.3)$ & & \\
\hline$>35$ & $5^{c}(13.2)$ & $3^{d}(11.5)$ & & & $6^{c}(14.6)$ & $2^{d}(8.7)$ & & \\
\hline \multicolumn{3}{|l|}{ Marital status } & 0.769 & 0.93 & & & 0.452 & 1.19 \\
\hline Married & $28^{a}(73.7)$ & $20^{b}(76.9)$ & & & $32^{\mathrm{a}}(78)$ & $16^{\mathrm{b}}(69.6)$ & & \\
\hline Unmarried & $10^{c}(26.3)$ & $6^{d}(23.1)$ & & & $9^{c}(22)$ & $7^{d}(30.4)$ & & \\
\hline Job status & & & 0.935 & 1.02 & & & 0.835 & 0.95 \\
\hline Doctor & $31^{\mathrm{a}}(81.6)$ & $21^{b}(80.8)$ & & & $33^{\mathrm{a}}(80.5)$ & $19^{b}(82.6)$ & & \\
\hline Other supporting staff & $7^{c}(18.4)$ & $5^{d}(19.2)$ & & & $8^{c}(19.5)$ & $4^{d}(17.4)$ & & \\
\hline \multicolumn{3}{|l|}{ Covid status } & 0.545 & 0.88 & & & 0.352 & 0.84 \\
\hline Positive (Exposed) & $19^{\mathrm{a}}(50)$ & $15^{\mathrm{b}}(57.7)$ & & & $20^{a}(48.8)$ & $14^{b}(60.9)$ & & \\
\hline Negative (unexposed) & $19^{c}(50)$ & $11^{d}(42.3)$ & & & $21^{c}(51.2)$ & $9^{d}(39.1)$ & & \\
\hline \multicolumn{3}{|l|}{ Gender } & 0.223 & - & & & 0.178 & - \\
\hline Female & $38^{\mathrm{a}}(100)$ & $25^{b}(96.2)$ & & & $41^{\mathrm{a}}(100)$ & $22^{b}(95.7)$ & & \\
\hline Male & $0^{c}$ & $1^{d}(3.8)$ & & & 0 & $1(4.3)$ & & \\
\hline \multicolumn{3}{|l|}{ Depression } & $<0.001$ & 0.27 & & & $<0.001$ & 2.82 \\
\hline Yes & $5^{\mathrm{a}}(13.2)$ & $18^{b}(69.2)$ & & & $33^{\mathrm{a}}(80.5)$ & $5^{\mathrm{b}}(21.7)$ & & \\
\hline No & $33^{c}(86.8)$ & $8^{d}(30.8)$ & & & $8^{c}(19.5)$ & $18^{\mathrm{d}}(78.3)$ & & \\
\hline \multicolumn{9}{|c|}{$\begin{array}{l}+ \text { Abbreviation Relative Risk, formula for } R R \text { used was [Equation]. a, } b, c, d \text { are the cell content for the calculation with above formulas. Incidence of anxiety } \\
\text { n COVID survivor HCWs }=[\text { Equation] }=19 / 19+15=0.56 . \text { Incidence of anxiety in non-COVID HCWs }=[\text { Equation] }=19 / 19+11=0.63 . \text { Relative risk for anxiety among } \\
\text { HCWs who survived COVID infection. RR }=[\text { Equation }]=0.56 / 0.63=0.88 . \text { Incidence of depression in COVID survivor HCWs }=[\text { Equation] 20/20+14 }=0.59 . \text { Incidence of } \\
\text { depression in non COVID HCWs }=[\text { Equation] }=21 / 21+9=0.70 . \text { Relative risk of developing depression among HCW who survived COVID infection. RR }=[\text { Equation] }= \\
0.59 / 0.70=0.84 .\end{array}$} \\
\hline
\end{tabular}

Ethical approval was obtained from LRH-Ethical Review Board in July 2020. Informed consent forms (ICF), were made both in English and Urdu for easy understanding of the participants. Any identifiers including names or medical record numbers for participants were removed from the data. Personal data were de-identified through codes. Information about basic sociodemographic along with anxiety and depression using the Hospital Anxiety Depression Scale (HADS) was gathered. The HADS instrument was administered by two medical doctors. Data were analysed using STATA Corp 15.1. (StataCorp, LLC, College Station, TX). Categorical variables were reported as frequencies and percentages. Anxiety and depression scores were reported as normal, borderline, and abnormal scores. The categories of borderline and abnormal scores were merged to avoid sparse data and for generating $2 \times 2$ tables for disease and exposure status. Categorical variables were compared using the Chi-square test. P-values for the two-tailed hypothesis were reported, with $p$ $<0.05$ considered significant. Relative risk was calculated manually.

Eighty-six participants were enrolled while 65 participants completed the paper-based HADS questionnaire. Sixty-three(98.6\%) participants were females; $84.4 \%(n=54)$ participants were $25-35$ years of age; $75 \%(n=48)$ were married. The HADS scores for the 64 study participants can be seen in Table I. Twenty-five percentage participants, who were initially enrolled in the study, did not complete the six-month evaluation, and were excluded from the analysis. The risk of anxiety and depression has been tabulated in Table I. An insignificant difference in the risk of anxiety among COVID-19 survivor HCWs was seen as compared to the COVID-19 negative participants. An insignificant difference in the risk of depression among COVID-19 survivor HCWs was seen as compared to the COVID-19 negative participants. The percentages of participants with borderline anxiety score (32.8\%), abnormal anxiety score (26.6\%), borderline depression score (28.1\%), and abnormal depression scores (35.9\%).

The present results did not show an increased risk of anxiety and depression among COVID-19 survivor HCWs, compared to the uninfected HCWs. Although longitudinal studies are lacking from Pakistan; however, international cross-sectional studies have been reported.

The presently reported percentages of abnormal anxiety score $(26.6 \%)$ and depression score $(35.9 \%)$ among healthcare workers were higher, compared to a study from China (anxiety $20.1 \%$ and depression $12.7 \%),{ }^{4}$ and India (anxiety $3.7 \%$ and depression $11.4 \%)^{5}$; but lower compared to United States (33\% for anxiety and $48 \%$ depression). ${ }^{6}$ 
When compared the findings with the study from Mazza et al., they reported $57 \%$ anxiety or depressive symptoms, which is higher than this study. ${ }^{2}$

There was no sufficient evidence to conclude that COVID-19 positive HCWs had a higher risk of anxiety or depression, compared to uninfected HCWs. However, this study provides important findings, upon which future longitudinal studies can be built. The main strength of this study was its cohort design and controls, selected from the same unit to limit confounding factors. Limitations include fewer male participants, a smallsample size, and $25 \%$ loss to follow-up. A baseline assessment of the participants with HADS was not done; hence, the undiagnosed baseline anxiety or depression cannot be ruled out.

\section{ETHICAL APPROVAL:}

Protocol was submitted to the LRH Ethical Review Board. Approval for the study was obtained in July 2020, reference No. 577/LRH/MTI.

\section{PATIENTS' CONSENT:}

Informed consent forms (ICF) were taken. Any identifiers including names or medical record numbers for participants were not included in data. Personal data was de-identified through codes.

\section{CONFLICT OF INTEREST:}

The authors declared no conflict of interest.

\section{AUTHORS' CONTRIBUTION:}

FZ: Conception, protocol development, ethical approval, and data collection and final approval of the version to be published.

ZN: Conception, study design, ethical approval and instruments selection.

SM: Study design, methodology, manuscript writing, data analysis and interpretation.
RR: Data collection and manuscript review.

All authors read the final draft and approved for publication.

\section{REFERENCES}

1. Fiorillo A, Gorwood P. The consequences of the COVID-19 pandemic on mental health and implications for clinical practice. Eur Psychiatry 2020; 63(1):e32. doi: 10.1192/ j.eurpsy.2020.35.

2. Mazza MG, De Lorenzo R, Conte C, Poletti S, Vai B, Bollettini I, et al. Anxiety and depression in COVID-19 survivors: Role of inflammatory and clinical predictors. Brain Behav Immun 2020; 89:594-600. doi: 10.1016/j.bbi.2020.07.037.

3. Sethi A, Aamir HS, Sethi BA, Ghani N, Saboor S. Impact on frontline nurses in the fight against coronavirus disease. Ann King Edward Med Uni 2020; 26(Special Issue):120-5.

4. Du J, Dong L, Wang T, Yuan C, Fu R, Zhang L, et al. Psychological symptoms among frontline healthcare workers during COVID-19 outbreak in Wuhan. Gen Hospital Psychiatry 2020; 67:144-5. doi: 10.1016/j.genhosppsych. 2020.03.011.

5. Wilson W, Raj JP, Rao S, Ghiya M, Nedungalaparambil $\mathrm{NM}$, Mundra $\mathrm{H}$, et al. Prevalence and predictors of stress, anxiety, and depression among healthcare workers managing COVID-19 pandemic in India: A nationwide observational study. Indian J Psychol Med 2020; 42(4): 353-8. doi: 10.1177/0253717620933992.

6. Shechter A, Diaz F, Moise N, Anstey DE, Ye S, Agarwal S, et al. Psychological distress, coping behaviors, and preferences for support among New York healthcare workers during the COVID-19 pandemic. Gen Hospital Psychiatry 2020; 66:1-8. doi: 10.1016/j.genhosppsych. 2020.06.007. 